\title{
ESTIMATIVA DO ÍNDICE DE ÁREA FOLIAR E TEMPERATURA EM UMA ÁREA DE EUCALIPTO
}

\author{
Raianny Leite do N. Wanderley ${ }^{1, *}$, Humberto R. da Rocha ${ }^{1}$, Osvaldo M. R. Cabral ${ }^{2}$, \\ Helber C. de Freitas ${ }^{1}$, Emília M. S. Brasilio ${ }^{1}$, Jonathan M. da Silva ${ }^{1}$ \\ ${ }^{1}$ Laboratório de Clima e Biosfera, IAG/USP \\ ${ }^{2}$ Embrapa Meio Ambiente \\ *raiannyln@yahoo.com.br
}

\begin{abstract}
It is known that the presence of large masses of vegetation is a factor that can influence the microclimate of a region. In this paper we analyzed the correlation between leaf area index (LAI) and land surface temperature (LST), both estimated from remote sensing images from Landsat-5 TM in an area of eucalyptus plantation, and these estimates were compared to the observed data. The correlation between LAI and LST was not significant (16\%), which indicates that there is no necessarily a direct influence of vegetation in the local temperature. The comparison between estimated and observed data shows that the application of remote sensing techniques in the estimative of interested variables is efficient, because the estimatives ollowed consistently the observed values.
\end{abstract}

Keywords: Remote Sensing, vegetation, climate.

\section{INTRODUÇÃO}

A presença de grandes massas de vegetação é um importante influenciador nas condições da temperatura local (Llandert, 1982 apud Alvarez, 2004). O índice de área foliar (IAF), definido pela razão entre a área foliar do dossel por unidade de área projetada no solo $\left(\mathrm{m}^{2} \mathrm{~m}^{-}\right.$ ${ }^{2}$ ), é um componente determinante do microclima, tanto abaixo do dossel quanto acima e pode ser obtido por diversos métodos (Gower et al, 1999), tanto por medidas diretas (fotografia hemisférica) como por estimativas (sensoriamento remoto), neste, indicando a biomassa de casa pixel da imagem (Allen et al., 2002). Sendo assim, neste trabalho foi estimado o IAF e a temperatura da superfície $\left(\mathrm{T}_{\mathrm{S}}\right)$ para três pontos em uma área de plantação de Eucalipto, com idades de 2 a 6 anos, durante março-setembro dos anos de 2006 e 2007. 
Também foi analisada qual a correlação entre essas duas variáveis e as estimativas foram comparadas com dados observados.

\section{MATERIAIS E MÉTODOS}

Os pontos estão localizados no município de Santa Rita do Passa Quatro-SP e foram escolhidos de acordo com idades e período de cortes diferentes para que se pudesse ter maior confiabilidade nos resultados estimados. Foram nomeados de acordo com sua idade (em anos) no ano de 2006, sendo assim: Euc-5A (-21.607, -47.602, 702m), Euc-3A (-21.586, -47.610, 751m) e Euc-2A (-21.601, -47.636, 720m). A estimativa do IAF e da $T_{S}$ foi realizada aplicando o algoritmo SEBAL (Bastiaanssen et al., 1998a,b) a partir de dados do sensor TM do satélite Landsat-5 (resoluções espacial: 30m e temporal: 16 dias). Os dados do IAF observados foram derivados de fotografias hemisféricas (Cabral et al., 2011) para Eucalipto de 2-4 anos de idade. Os dados da temperatura do ar $\left(\mathrm{T}_{\mathrm{AR}}\right)$ foram obtidos com o sensor HMP45C (instalado acima do dossel e mede $\mathrm{T}_{\mathrm{AR}}$ e umidade relativa) e foi utilizada neste trabalho como valor de referência para analisar a $\mathrm{T}_{\mathrm{S}}$ estimada.

\section{RESULTADOS E DISCUSSÃO}

Na Figura 1, que apresenta a distribuição do IAF (estimado e observado), foi notada uma concentração entre 2,0 e 3,3. Os menores valores estimados foram no ponto onde o Eucalipto estava com 5 anos, com mínimo e máximo de 1,83 e 2,82, respectivamente e os maiores valores foram para o Eucalipto com 3 anos de idade, com mínimo de 2,16 e máximo de 3,26. A média dos valores estimados foi 2,6. Os valores mínimos e máximos observados em campo foram 2,23 e 3,05, respectivamente, e média de 2,74. Então, o IAF estimado a partir de imagens do sensor TM subestimou em média 5\% o IAF observado, o que pode ser atribuído à resolução espacial do sensor, pois o valor representado no pixel corresponde ao valor predominante em tal. 


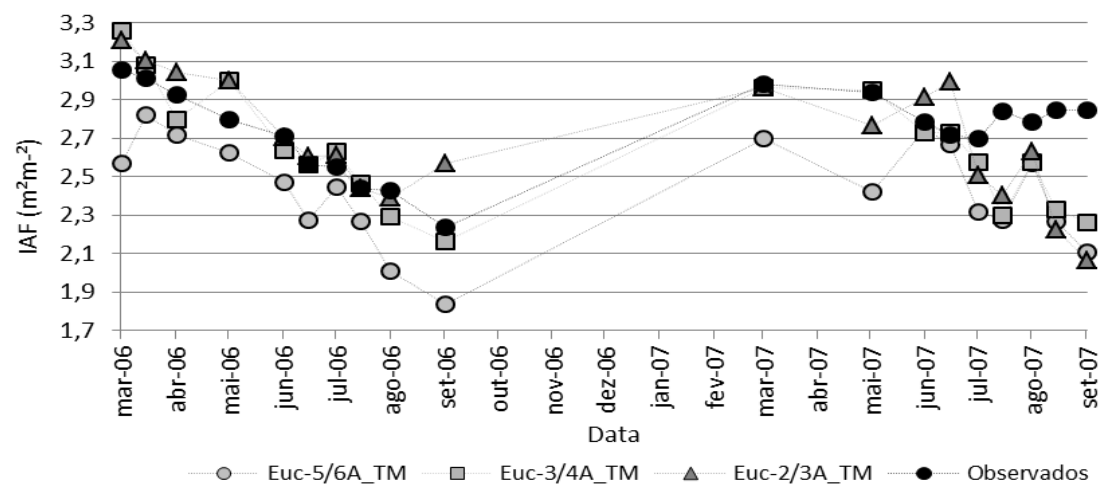

Figura 1: Índice de área foliar observado (foto hemisférica) e estimado (sensor TM) para o Eucalipto com idades de 2-6 anos em Santa Rita do Passa Quatro-SP.

A Figura 2 apresenta os valores da $\mathrm{T}_{\mathrm{S}}$ estimada, bem como a $\mathrm{T}_{\mathrm{AR}}$ observada. Os valores mínimos e máximos estimados para os pontos foram em média $11,5^{\circ} \mathrm{C}$ e $23,5^{\circ} \mathrm{C}$, respectivamente, e média geral de $19,5^{\circ} \mathrm{C}$. A $\mathrm{T}_{\mathrm{AR}}$ mínima observada foi $12^{\circ} \mathrm{C}$, a máxima $23,5^{\circ} \mathrm{C}$ e a média foi $19,6^{\circ} \mathrm{C}$. Houve uma pequena subestimativa dos valores estimados em relação aos observados, cerca de $1,7 \%$, que pode ser atribuída tanto à resolução espacial como também ao fato de que a temperatura observada é a temperatura do ar. Também podem ser observadas duas bruscas quedas de temperatura nos meses de setembro/06 e junho/07, que se deve ao fato de que nesses dias houve registro de chuva.

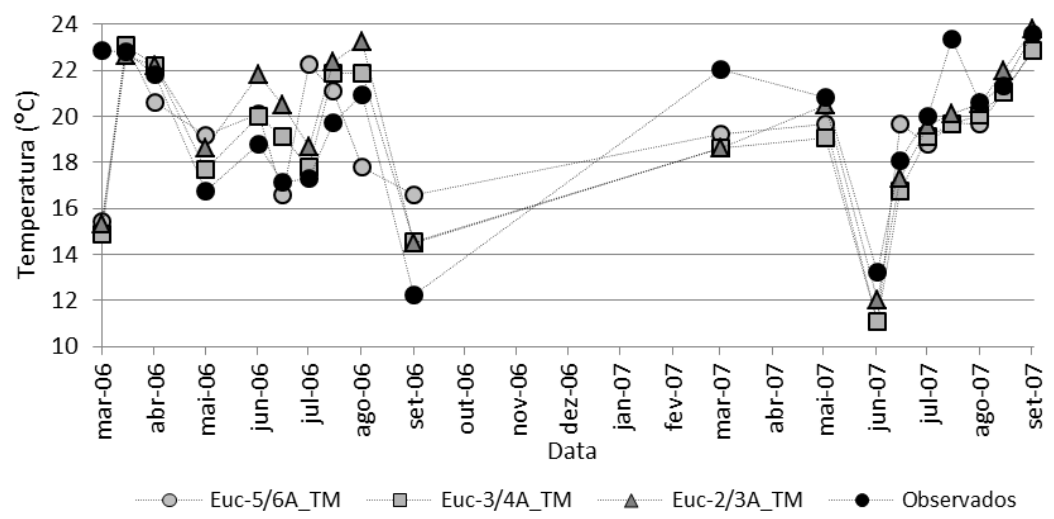

Figura 2: Temperatura da superfície estimada (sensor TM) e temperatura do ar observada (HMP45C) para o Eucalipto com idades de 2-6 anos em Santa Rita do Passa Quatro-SP.

Neste caso, a presença de uma plantação de Eucalipto na região não foi um fator significativo na variação da temperatura local, pois apresentou correlação média de 0,16. 


\section{CONCLUSÕES}

A comparação entre os valores estimados pelo SEBAL e os dados observados mostra que a aplicação de técnicas de sensoriamento remoto na estimativa das variáveis estudadas é eficiente, pois os valores estimados acompanharam de forma coerente os valores observados. É importante que a estimativa do IAF e da $\mathrm{T}_{\mathrm{S}}$ feita por técnicas sensoriamento remoto seja comparada aos dados de campo para que possa ser garantida uma coerência com a realidade. Outros tipos de coberturas vegetais serão analisados, bem como outros índices de vegetação serão estimados.

\section{REFERÊNCIAS}

ALLEN, R. G., TREZZA, R. \& TASUMI M. Surface energy balance algorithms for land. Advance training and user's manual, version 1.0, pp.98, 2002.

ALVAREZ, I. A. Qualidade do espaço verde urbano: uma proposta de índice de avaliação. Tese (doutorado) - Escola Superior de Agricultura Luiz de Queiroz, 2004.

BASTIAANSSEN, W. G. M.; MENENTI, M.; FEDDES, R. A. \& HOLTSLAG, A. A. M. A. Remote Sensing Surface Energy Balance Algorithm for Land (SEBAL) 1. Formulation. Journal of Hydrology, v. 212-213, p. 198-212, 1998a.

BASTIAANSSEN, W. G. M., PELGRUM H., WANG, J. MORENO, Y. M.J., ROERINK, G. J. \& VAN DER WAL, T. The Surface Energy Balance Algorithm for Land (SEBAL) 2. Validation. Journal of Hydrology, 212-213: 213-229, 1998 b.

CABRAL, O. M. R., GASH, J. H. C., ROCHA, H. R., MARSDEN, C., LIGO, M. A. V., FREITAS, H. C., TATSCH, J. D., GOMES, E. Fluxes of $\mathrm{CO} 2$ above a plantation of Eucalyptus in southeast Brazil. Agric. Forest Meteorol., 151, p. 49-59, 2011.

GOWER. S. T., KUCHARIK C. J., NORMAN J. M. Direct and indirect estimation of leaf area index, $f_{A P A R}$, and net primary production of terrestrial ecosystems. Remote Sensing of Environment, v.70, p.29-51, 1999. 